\title{
ATRASO NO DESENVOLVIMENTO NEUROPSICOMOTOR E DEFICIÊNCIA INTELECTUAL: AVALIAÇÃO DO NÚMERO DE CASOS ATENDIDOS EM UM CENTRO DE ESPECIALIDADES MÉDICAS DE BELÉM - PA
}

\section{ARTIGO ORIGINAL}

EL-HUSNY, Antonette Souto ${ }^{1}$

DIAS, Amanda de Araújo ${ }^{2}$

MARTINS, Bianca Azevedo Parreira ${ }^{3}$

BORGES, Samuel Maurício Ferreira ${ }^{4}$

MOREIRA, Elisângela Claudia de Medeiros ${ }^{5}$

DENDASCK, Carla Viana ${ }^{6}$

${ }^{1}$ Doutora em Genética. Médica. Docente e Pesquisadora do Centro Universitário do Estado do Pará (CESUPA).

${ }^{2}$ Acadêmica de Medicina do Centro Universitário do Estado do Pará (CESUPA).

${ }^{3}$ Acadêmica de Medicina do Centro Universitário do Estado do Pará (CESUPA).

${ }^{4}$ Acadêmico de Medicina do Centro Universitário do Estado do Pará (CESUPA).

${ }^{5}$ Mestre em Teoria e Pesquisa do Comportamento. Docente da Universidade do Estado do Pará - UEPA. Doutoranda em Medicina/Doenças Tropicais na Universidade Federal do Pará - NMT/UFPA.

${ }^{6}$ Teóloga. Doutora em Psicanálise Clínica. Pesquisadora do Centro de Pesquisa e Estudos Avançados, São Paulo, SP. 
OLIVEIRA, Ciane Martins de ${ }^{7}$

OLIVEIRA, Euzébio de ${ }^{8}$

EL-HUSNY, Antonette Souto. Et al. Atraso no desenvolvimento Neuropsicomotor e Deficiência Intelectual: avaliação do número de casos atendidos em um centro de especialidades médicas de Belém - PA. Revista Científica Multidisciplinar Núcleo do Conhecimento. Ano 04, Ed. 12, Vol. 07, pp. 17-23. Dezembro de 2019. ISSN: 2448-0959, Link de acesso: https://www. nucleodoconhecimento.com.br/saude/desenvolvimento-

\section{neuropsicomotor}

\section{RESUMO}

Introdução: O Déficit Intelectual (DI) e o Atraso no Desenvolvimento Neuropsicomotor (ADNPM) são motivos comuns de atendimentos em ambulatórios de genética clínica. Objetivo: Identificar a proporção de casos atendidos no ambulatório de Genética clínica do Centro de Especialidades Médicas (CEMEC) encaminhados por Atraso no Desenvolvimento Neuropsicomotor e/ou Deficiência Intelectual. Metodologia: Foram agrupados os casos encaminhados por ADNPM e DI e subdivididos em grupos com diagnóstico já estabelecido e os casos sob investigação do diagnóstico. Resultados: Os atendimentos motivados por ADNPM ou DI representaram 46\% (33/71) das consultas do ambulatório médico. Destes, apenas $15 \%$ (5/33) dos casos já possuíam diagnóstico genético estabelecido. Dois casos avaliados foram concluídos como provável etiologia não genética e a maioria dos casos, $78 \%$ (26/33), permanecem em investigação. Considerações finais: A

7 Doutora em Ciências Biológicas. Docente e Pesquisadora do Centro Universitário do Estado do Pará (CESUPA).

${ }^{8}$ Doutor em Medicina/Doenças Tropicais. Docente e Pesquisador na Universidade Federal do Pará - UFPA. Pesquisador Colaborador do Núcleo de Medicina Tropical NMT/UFPA. 
identificação desses casos é extremamente importante para a saúde coletiva, bem como para que haja maiores investimentos na área de métodos terapêuticos, da genética médica e demais métodos de diagnóstico para a ADNPM.

Descritores: Genética, hereditariedade, deficiência intelectual, deficiências do desenvolvimento.

\section{INTRODUÇÃO}

O aumento populacional, têm provocado várias mudanças no panorama brasileiro das doenças da infância, bem como o aumento na sua detecção, e o surgimento de maior número de casos de doenças crônico-degenerativas e suas complicações e comorbidades (VICTORA; AQUINO, 2011).

O fato do acontecimento no Atraso Global no Desenvolvimento (AGD) e a Deficiência Intelectual (DI) tem surgido como situações de atendimento pediátrico considerados quase que rotineiros, atingindo até cerca de 3\% da população, decorrentes de uma variedade de causas, muitas destas, de origem genéticas em seu surgimento e com consequências no manuseio e do tratamento e atendimento da criança, incluindo sua família (SROUR; SHEVELL, 2014). Vale destacar que o termo "deficiência intelectual suplantou o termo retardo mental, que deve ser evitado por ser considerado pejorativo". Em geral, em tais situações, o pediatra é o primeiro profissional a lidar diretamente com estes pacientes e seus familiares (BOY, 2016, p. 177).

O Déficit Intelectual (DI) e Atraso no Desenvolvimento Neuropsicomotor (ADNPM) são motivos comuns de atendimentos em ambulatórios de genética clínica. No entanto, com a melhoria dos serviços de saúde, as causas genéticas tendem a tornarem-se proporcionalmente maiores do que alterações orgânicas adquiridas (PINA NETO et al., 1997).

Com o aumento na demanda por serviços médicos especializados para essas doenças, o ambulatório de Genética do Centro de Especialidades Médicas (CEMEC) 
do Centro Universitário do Estado do Pará (CESUPA) oferece possibilidade de ampliar a investigação diagnóstica do ADNPM e DI referentes a doenças genéticas.

Diante desse contexto o objetivo desse estudo de caso foi identificar a proporção de casos atendidos no ambulatório de Genética do Centro de Especialidades Médicas (CEMEC) encaminhados por suspeita diagnóstica de ADNPM e DI.

\section{METODOLOGIA}

Foi realizado um estudo longitudinal e descritivo, no qual foram coletados dados de 71 prontuários médicos dos pacientes atendidos no ambulatório de Genética do Centro de Especialidades Médicas (CEMEC) do Centro Universitário do Estado do Pará (CESUPA), do período de 1 de agosto de 2014 a 20 de dezembro de 2018. Estes atendimentos foram agrupados em casos encaminhados por ADNPM e DI e subdivididos em grupos com diagnóstico estabelecido e os casos com diagnóstico sob investigação. Cabe destacar que todos os pacientes assinaram um termo de autorização para a realização da pesquisa, utilizando seus dados clínicos contidos em seus prontuários médicos.

\section{RESULTADOS E DISCUSSÃO}

Os atendimentos motivados por ADNPM ou DI representaram $46 \%$ (33/71) das consultas realizadas no ambulatório. (Figura 1). 
Figura 1 - Casos atendidos no Ambulatório de Genética do CEMEC de ADNPM e outros.

\section{- ADNPM 33 - Outras causas 38}
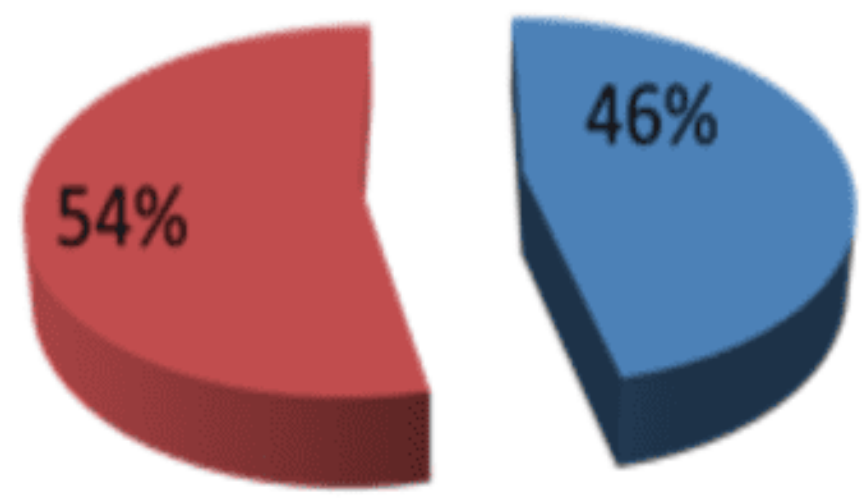

Fonte: Dados da própria pesquisa.

Destes, apenas 15\% (5/33) dos casos possuíam diagnóstico genético estabelecido; dois casos avaliados, representando $6 \%$ foram concluídos como provável etiologia não genética e a maioria dos casos, $78 \%$ (26/33), permanecem em investigação, pois necessitavam de exames que comprovassem ou não a doença. 
Figura 2 - Causas de Atraso no Desenvolvimento Neuropsicomotor em pacientes atendidos no ambulatório de Genética do Centro de Especialidades Médicas (CEMEC) do Centro Universitário do Estado do Pará (CESUPA).

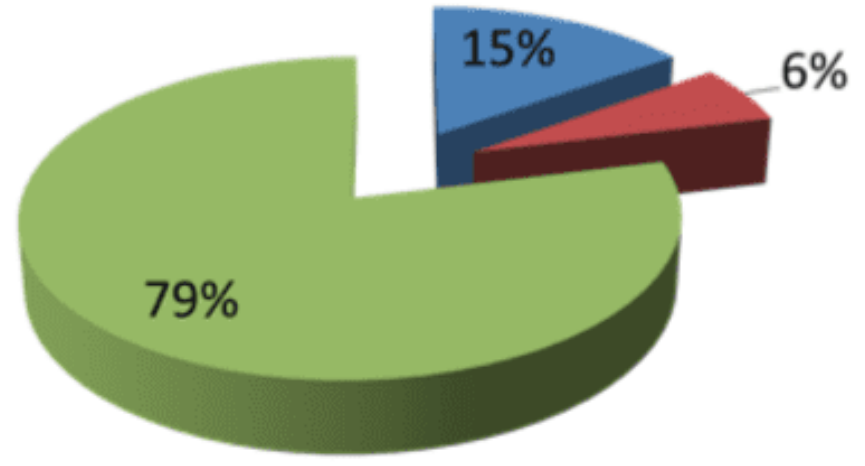

- Diagnóstico Genético

- Provável Etiologia Não Genética Em Investigação

Fonte: Dados da própria pesquisa.

Dentre os casos que possuíam diagnóstico genético confirmado para ADNPM e DI, predominaram as Alterações cromossômicas diversas (4 casos); Deleção parcial em 2q (1); Material adicional em 22p (1); Síndrome de Williams (1); Material adicional em $18 q$ (1). Um caso foi associado a doença monogênica reconhecível clinicamente (Acondroplasia), não sendo esta última comumente associada a ADNPM e DI.

A anamnese e o exame físico com ênfase em características morfológicas constituem a base fundamental para os estudos da etiologia da ADNPM e DI, uma vez que, acoplados ao exame neurológico podem alcançar chance de detecção diagnóstica de até $80 \%$ dos casos (KARNEBEEK; JANSWEIJER, 2005). A anamnese deve ser obtida da forma mais completa possível, de preferência com os pais ou cuidadores mais próximos da criança, atentando-se para dados específicos descritos a seguir (BOY SILVA; VASCONCELOS; PAIVA, 2014).

A importância do diagnóstico correto da ADNPM e DI, embora difícil, é justificada pelos benefícios associados, que ultrapassam a eventualidade da cura, em especial quando realizados precocemente (MOESCHLER; SHEVELL; COMMITTEE ON GENETICS, 2014; BOY, 2016). 
A partir deste esclarecimento etiológico, na existência de causas genéticas, a oferta do aconselhamento genético $(A G)$ pode ser provida pelo médico geneticista clínico. Vale sinalizar que o AG é um procedimento informativo que lida não apenas com "o diagnóstico, prognóstico, riscos de ocorrência genética e dos riscos de recorrência, mas também com orientações para a família e uso de medidas diagnósticas préconcepcionais e pré-natais". Dessa forma, faz-se necessária a adequada abordagem diagnóstica, e esta demandará trabalho multidisciplinar (BOY, 2016, p. 178).

\section{CONCLUSÃO}

Com base nos resultados deste estudo e possível concluir que a presença de casos de ADNPM e DI são expressivos no ambulatório de genética estudado. Porém, a capacidade diagnóstica é, em parte, limitada a cromossomopatias, o que representa limitação de acesso a outros métodos diagnósticos. Isso se reflete pela falta de recursos relacionadas a testes genéticos para discriminação de síndromes e patologias de difícil descoberta. Entretanto, identificar esses casos é extremamente importante para que haja maiores investimentos na área da genética médica e métodos terapêuticos e diagnósticos.

\section{REFERÊNCIAS}

BOY SILVA, R.T.; VASCONCELOS, M. M.; PAIVA, I. S. A criança com deficiência intelectual - aspectos genéticos, diagnósticos e terapêuticos. In: Campos Jr D.; BURNS, A. R. B.; LOPES, F. A. org. Tratado de Pediatria. São Paulo: Ed. Manole, 2014. p.1315-22.

BOY, R. Abordagem diagnóstica de crianças com atraso do desenvolvimento e deficiência intellectual. Revista HUPE, Rio de Janeiro;15(2): p. 177-186, 2016. DOI: 10.12957/rhupe. 2016. 28244.

CHEN, I.C. et al. Clinical analysis of 1048 children with developmental delay. Chang Gung Med J, 25: p. 743-750, 2002. 
KARNEBEEK, C. D. M.; JANSWEIJER, M. C. E. Diagnostic investigations in individuals with mental retardation: a systematic literature review of their usefulness. European Journal of Human Genetics. 13: p. 6-25, 2005.

KOUL, R.; AL-YAHMEDY, M.; AL-FUTAISI, A. Evaluation of children with global developmental delay: a prospective study at Sultan Qaboos University Hospital. Oman Med J. 27: p. 310-3, 2012.

MENEZES, T. O.; SMITH, C. A.; PASSOS, L. T.; PINHEIRO, H. H., MENEZES, S. A. Profile of special needs patients at a pediatric dentistry clinic. RBPS. 24: p.136-41, 2011.

MOESCHLER, J. B.; SHEVELL, M. AND COMMITTEE ON GENETICS. Comprehensive Evaluation of the Child With Intellectual Disability or Global Developmental Delays. Pediatrics, p. 134 e 903, 2014.

PINA NETO, J. M.; FERRAZ, V. E.; MOLFETTA, G. A., BUXTON, J.; RICHARDS, S. Clinicalneurologic, cytogenic and molecular aspects of the Prader-Willi and Angelman syndromes. Arq Neuropsiquiatr. 55: p. 199-208, 1997.

SROUR, M.; SHEVELL, M. Genetics and the investigation of developmental delay/intellectual disability. Arch Dis Child, Apr; 99 (4), p. 386-389, 2014.

VICTORA, C. G.; AQUINO, E. M. L. Maternal and child health in Brazil: progress and challenges; Lancet, 377: p. 1863-1876, 2011.

WILLIAMS, J. Global developmental delay-globally helpful? Dev Med Child Neurol, 57: p. 227, 2010.

Enviado: Dezembro, 2019.

Aprovado: Dezembro, 2019. 